\title{
Undifferentiated carcinoma of the mesentery in a 51 year old male: A Case Report
}

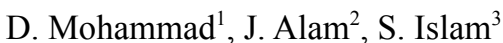

\begin{abstract}
Undifferentiated carcinoma is a usually aggressive, malignant epithelial neoplasm composed of atypical cells which do not display evidence of glandular, squamous, or urothelial cell differentiation.1 Mesenteric tumors are rare and consist of a heterogeneous group of lesions. Among them undifferentiated carcinoma of the mesentery is very very rare entity. Here we present a case of undifferentiated carcinoma of the mesentery in a 51 year old male admitted to the department of surgery, Shaheed Suhrawardy Medical College Hospital (ShSMCH) with rapidly increasing huge abdominal lump, central abdominal pain and weight loss. Ultrasound of the whole abdomen and CT scan of whole abdomen reports were suggestive of retroperitoneal mass. Exploratory laparotomy was done and specimen sent for histopathology. Reports were suggestive of malignant mesothelioma whereas immunohistochemistry report revealed undifferentiated carcinoma of the mesentery. To the best of our knowledge, there was no report of undifferentiated carcinoma of the mesentery in a 51 year old male from Bangladesh or any other country, 2013;5(2):114-116]
\end{abstract}

Keywords: undifferentiated carcinoma, the mesentery, mesothelioma

Received: August 2012; Revised: October 2013; Accepted: November 2013

\section{Introduction}

Mesenteric tumors are uncommon lesions that are generally considered inclusive of similar lesions of the omentum $^{4}$. These lesions may be cystic or solid, and they may demonstrate malignant or benign clinical behavior. No known etiologic or associated diseases have been reported in cases of primary mesenteric neoplasms. Solid primary tumors of the mesentery are rare. Published reports have consisted of small numbers of cases, which makes it difficult to determine the incidence of specific tumor types. Reasonable estimates of incidence range from 1 case per 200,000-350,000 population. Mesenteric tumors have been described as cystic in $40-60 \%$ of cases ${ }^{5}$. Malignant primary mesenteric tumors are extremely uncommon, even compared with primary malignancies of the small bowel. Approximately two thirds of malignant mesenteric tumors are mesenchymatous (most characterized as leiomyosarcoma or liposarcoma), while the remainder are primarily lymphomas ${ }^{6,7}$.

We present a case of a 51 years old man with undifferentiated carcinoma of the mesentery presenting with a huge abdominal mass with central abdominal pain \& weight loss, the diagnosis being made after immunohistochemistry following surgery, the first reported case in Bangladesh.

\section{Case Presentation}

A 51 year old male got admitted into Shaheed Suhrawardy Hospital under the department of Surgery, with the complaints of rapidly growing lump in the central abdomen for 3 months, dull aching central abdominal pain for 2

1. Dr. Din Muhammad, Assistant Professor of Surgery, Shaheed Suhrawardy Medical College, Hospital, Dhaka.

2. Dr. Jahangir Alam, Associate Professor of Surgery, Shaheed Suhrawardy Medical College \& Hospital, Dhaka

3. Dr. Sharmin Islam, Medical Officer, Casualty Surgery, Dhaka Medical College, Hospital, Dhaka.

\section{Correspondence}

Dr. Din Muhammad, FCPS (Surgery), Assistant Professor, Surgery, Shaheed Suhrawardy Medical College, Hospital, Dhaka. Email: dr.din_zip@yahoo.com 
months, significant weight loss $(5 \mathrm{~kg})$ within last 2 months. (photograph $1 \& 2$ )

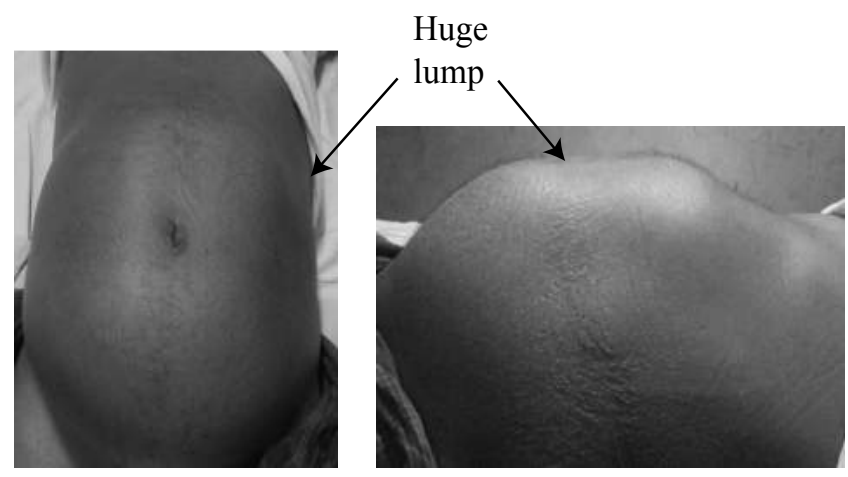

Photograph $1 \& 2$ : Huge abdominal lump

There was no history of fever, anorexia, vomiting, alteration bowel habit, hematemesis, melaena, hematochezia or hematuria. On examination, the patient was anxious looking, with average nutritional status (BMI $\left.23.55 \mathrm{~kg} / \mathrm{m}^{2}\right)$ \& was moderately anaemic with no lymphadenopathy. His vital parameters were within normal limit. Abdomen was distended, flanks were full. Umbilicus was centrally placed \& vertically slit. There was an intra-abdominal lump approx. 20x12 cm in size and occupying hypogastric, both illiac, lumber, umbilical and part of epigastric region. The lump was variegated in consistency and mildly tender. Surface of the lump was irregular with ill defined margin but upper and lower limit could be reached. The lump was non pulsatile, slightly mobile from side to side, but not above downward and did not move with respiration. There was no other organomegaly, no ascites. Digital rectal examination revealed no abnormality. All other systemic examination findings were within normal limits.

- On routine blood count, $\mathrm{Hb} \%$ was $8.7 \mathrm{gm} / \mathrm{dl}$ and ESR was $56 \mathrm{~mm}$ in 1st hr. Ultrasound of whole abdomen revealed a mixed echogenic mass lesion measuring about $15 \times 11.3 \mathrm{~cm}$ is seen in pre-vertebral region at the level of umbilicus, compressing the abdominal aorta with mild ascites suggestive of retroperitoneal mass.

- CT scan of abdomen showed A large well defined almost rounded mixed density mass lesion measuring about $19.9 \mathrm{X} 11.2 \mathrm{~cm}$ is noted in abdomino-pelvic cavity. Cranio-caudal extension of the mass is seen from L1 to S1 vertebra. After IV contrast mild heterogenous enhancement of the mass is seen. Abdominal aorta \& IVC are compressed by the mass. Bowel loops are splayed upwards $\&$ laterally, suggestive of retroperitoneal mass (photograph $3,4)$

- Photograph 3 \& 4: CT scan of the abdomen showing huge mixed density mass lesion.

Exploratory laparotomy was done under general anaesthesia with endotracheal intubation. With all aseptic precaution, abdomen was opened by midline incision. A large mass of approx.20X15 cm in size was found arising
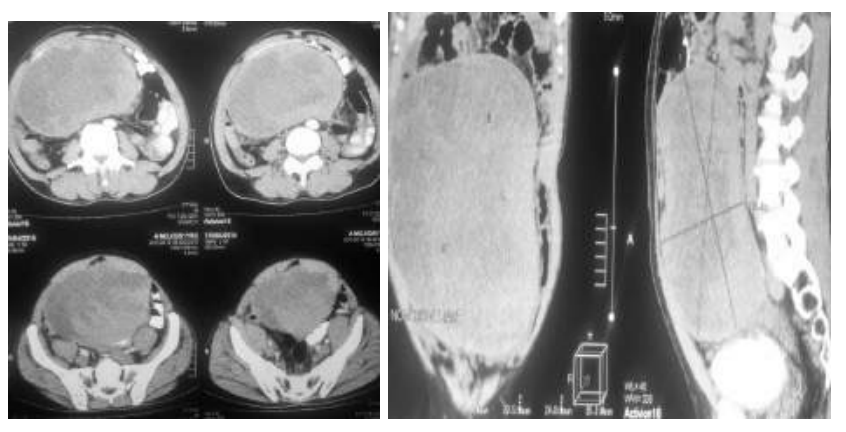

from 'The Mesentery' attached to Jejunum . The mass was partly cystic, partly solid; solid component composed of brown friable tissue (photograph 5,6,7). Liver was normal and there was no ascites. Tumour mass and adjacant jejunum was resected completely keeping the healthy margin. Continuation of gut was maintained by end to end anastomosis. After proper haemostatis, abdomen was closed in layers. A drain tube was left in pelvic region. 2units of whole blood was transfused per-operatively.

- Postoperative recovery was uneventful. Histopathology report revealed Mesenteric tumour mass-consistent with malignant mesothelioma. Histopathogical slides were sent to another centre for review \& the reports revealed undifferentiated malignant neoplasm with areas of haemorrhage and necrosis infiltrating into the fibromascular tissue.
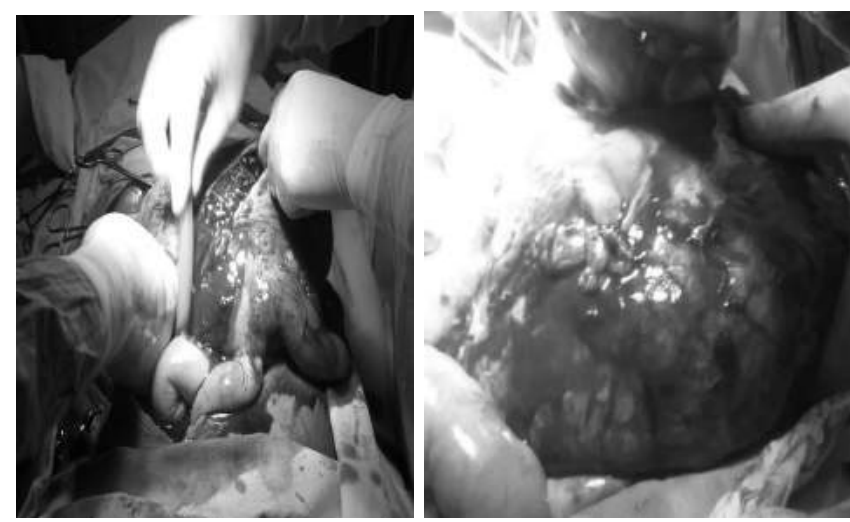

Photograph 5\&6 : Peroperative view of the tumour mass.

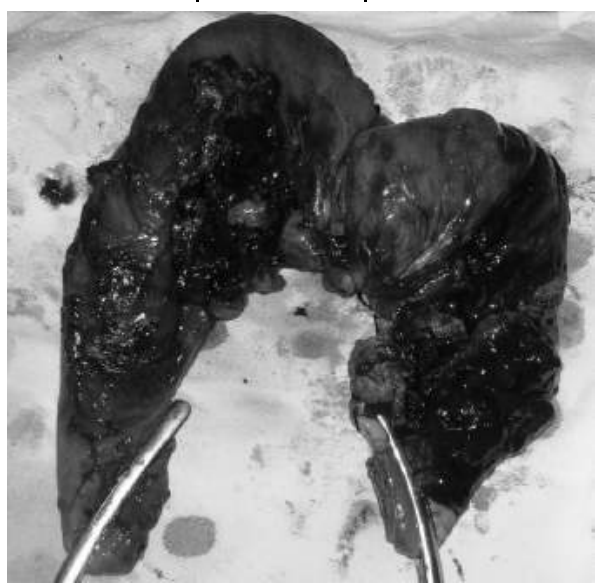

Photograph 7: The resected specimen 
- On immunohistochemistry, itws found that among the biomarker expression profiles, only Pan-cytokeratin (Clone AE/AE3) was found Positive. So Impression was Undifferentiated carcinoma.

- Chemotherapy was given according to the oncological consultation. Patient is under our regular follow up for last 6 months and found healthy.

\section{Discussion}

Mesenteric tumors are rare and consist of a heterogeneous group of lesions. Masses may arise from any of the mesenteric components: peritoneum, lymphatic tissue, fat, and connective tissue2. Although uncommon, mesenteric tumors are encountered in all age groups from infancy to the very elderly. These tumors should be considered as an explanation for a palpable abdominal mass, but they are most commonly brought into the differential diagnosis of abdominal pathology once a suggestive radiologic study or an abdominal operation has been performed. An increased awareness of neoplastic and non neoplastic processes that result in mesenteric masses aids the clinician in recognizing these diseases. Solid primary tumors of the mesentery are rare. Published reports have consisted of small numbers of cases, which makes it difficult to determine the incidence of specific tumor types.

Malignant primary mesenteric tumors are extremely uncommon, even compared with primary malignancies of the small bowel. Published reports suggest that one third to one half of all mesenteric masses are malignant tumors. The largest case series have been from France and China6,7. These reports indicate that approximately two thirds of malignant mesenteric tumors are mesenchymatous (most characterized as leiomyosarcoma or liposarcoma), while the remainder are primarily lymphomas. But undifferentiated carcinoma of the mesentery is a very rare entity. Here we presenting a case of undifferentiated carcinoma of the mesentery, which is to the best of our knowledge, 1 st reported case in Banglaesh, even throughout the world.

Clinical findings and symptoms associated with mesenteric tumors of all types are related to the presence of a mass lesion. Because the mass does not involve the tubular portion of the GI tract per se, obstructive symptoms are generally late findings in malignant mesenteric tumors and large benign tumors. Pain is the principal presenting symptom resulting in the discovery of a mesenteric mass. A palpable mass may also be present, although generally not without some abdominal pain. Nausea, vomiting, diarrhea, bloating, and constipation have also been described with mesenteric tumors.

Although most symptoms reflect a fairly indolent process, intestinal obstruction has been reported with benign and malignant mesenteric tumors. In the case of malignant tumors, this is generally secondary to aggressive local growth. Patients with mesenteric tumors exhibit signs and symptoms of intestinal obstruction; however, in contrast to primary tumors of the intestine, much bulkier disease may be present before obstructive findings are encountered. Computed tomography (CT) scan of a mesenteric stromal tumor. This is an infiltrative lesion surrounding vascular structures within the proximal jejunal mesentery. But in our case, preoperative investigations failed to reach the diagnosis. Therefore exploratory laparotomy was done and resected specimen was sent for histopatho;ogy. Final diagnosis was made after immunohistochemistry.

Characterization of protein markers of this tumor type has indicated that they are phenotypically very similar to the GIST group and are less frequently phenotypically related to retroperitoneal leiomyosarcomas. The absence of a detectable primary intestinal tumor signifies a primary mesenteric origin of the lesion. In our case, only Pan-cytokeratin(Clone AE/AE3) marker was found positive, therefore report was suggestive of undifferentiated carcinoma of the mesentery.

\section{Conclusion}

In conclusion, we have presented a very very rare case of undifferentiated carcinoma of the mesentery in a 51 year old male. Undifferentiated carcinoma of the mesentery is a very rare entity among the primary malignant tumours of the mesentery. In our case, although patient came with a common presentation of abdominal lump, dullaching pain and weight loss, all modalities of modern investigations were suggestive of retoperitoneal mass but the final diagnosis could be made following exploratory laparotomy , histopathology and immunohistochemistry. So again abdomen - the magic box showed its another magic when laparotomy was done. But proper history, clinical examination and proper knowledge as well in interpreting the imaging findings have no alternative for diagnosis such cases.

\section{References}

1. Bosman FT, Carneiro F, Hruban RH, Theise ND (Eds.).WHO Classification of Tumours of the Digestive System, 4th Edition, International Agency for Research on Cancer, 2010-7. journals.lww.com/.../Undifferent...www.pubcan.org

2. Dufay C, Abdelli A, Le Pennec V, Chiche L; Mesenteric tumors: diagnosis and treatment. J Visc Surg. 2012 Aug;149(4):239-51. doi:10.1016/j.jviscsurg.2012.05.005. Epub 2012 Jul 15.

3. Goh BK, Chow PK, Kesavan SM, et al. A single-institution experience with eight CD117-positive primary extragastrointestinal stromal tumors: critical appraisal and a comparison with their gastrointestinal counterparts. J Gastrointest Surg. 2009 Jun. 13(6):1094-8.

4. Mesenteric Tumors. Author: Neal E Seymour, MD; Chief Editor: John Geibel, MD, DSc, MSc, MA. Website: http://emedicine.medscape.com/article/191675

5. Metaxas G, Tangalos A, Pappa P, et al. Mucinous cystic neoplasms of the mesentery: a case report and review of the literature. World J Surg Oncol. 2009 May 19. 7:47.

6. Guivarc"h M. [Tumors of the mesentery. Apropos of 102 cases]. Ann Chir. 1994. 48(1):7-16.

7. Luo HS. [Mesentery tumor--clinico-pathologic analysis of 171 cases]. Chung Hua Chung Liu Tsa Chih. 1989 Mar. 11(2):139-41. 